IJMMS 25:7 (2001) 467-478

PII. S0161171201002381

http://ijmms.hindawi.com

(C) Hindawi Publishing Corp.

\title{
ON HENSTOCK-DUNFORD AND HENSTOCK-PETTIS INTEGRALS
}

\author{
YE GUOJU and AN TIANQING
}

(Received 22 October 1998)

\begin{abstract}
We give the Riemann-type extensions of Dunford integral and Pettis integral, Henstock-Dunford integral and Henstock-Pettis integral. We discuss the relationships between the Henstock-Dunford integral and Dunford integral, Henstock-Pettis integral and Pettis integral. We prove the Harnack extension theorems and the convergence theorems for Henstock-Dunford and Henstock-Pettis integrals.
\end{abstract}

2000 Mathematics Subject Classification. Primary 26A39, 28B05; Secondary 28B20, 46G10, $46 \mathrm{G} 12$.

1. Introduction. During 1957-1958, R. Henstock and J. Kurzweil, independently, gave a Riemann-type integral called the Henstock-Kurzweil integral (or Henstock integral) (see [7]). It is a kind of nonabsolute integral and contains the Lebesgue integral. It has been proved that this integral is equivalent to the special Denjoy integral [7]. The Dunford, Pettis integrals are generalizations of the Lebesgue integral to Banachvalued functions. In [5], R. A. Gordon gave two Denjoy-type extensions of the Dunford, Pettis integrals, the Denjoy-Dunford and Denjoy-Pettis integrals, and discussed their properties.

In this paper, we give the Riemann-type extensions of Dunford, Pettis integrals, the Henstock-Dunford, Henstock-Pettis integrals, and discuss the relationships between the Henstock-Dunford integral and Dunford integral, Henstock-Pettis integral and Pettis integral. We prove the Harnack extension theorems and the convergence theorems for Henstock-Dunford and Henstock-Pettis integrals.

Throughout this paper, $X$ denotes a real Banach space and $X^{*}$ its dual. $B\left(X^{*}\right)=$ $\left\{x^{*} \in X^{*}:\left\|x^{*}\right\| \leq 1\right\}$ is the unit ball in $X^{*} . I_{0}=[a, b]$ is a closed interval in $\mathbb{R}$.

We first give some preliminaries. A partition $D$ of $[a, b]$ is a finite collection of interval-point pairs $(I, t)$ with the intervals nonoverlapping and their union $[a, b]$. Here $t$ is the associated point of $I$. We write $D=\{(I, t)\}$, it is said to be $\delta$-fine partition of $[a, b]$ if for each interval-point pair $(I, t)$, we have $t \in I \subset(t-\delta(t), t+\delta(t))$.

DEFINITION 1.1 (see [7]). A function $f:[a, b] \rightarrow \mathbb{R}$ is Henstock integrable if there exists a function $F:[a, b] \rightarrow \mathbb{R}$ such that for every $\epsilon>0$ there is a function $\delta(t)>0$ such that for any $\delta$-fine partition $D=\{[u, v] ; t\}$ of $[a, b]$, we have

$$
\left|\sum[f(t)(v-u)-F(u, v)]\right|<\epsilon,
$$

where the sum $\sum$ is understood to be over $D=\{([u, v], t)\}$ and $F(u, v)=F(v)-F(u)$. We write $(H) \int_{I_{0}} f=F\left(I_{0}\right)$. 
The function $f$ is said to be Henstock integrable on the set $E \subset[a, b]$ if the function $f \chi_{E}$ is Henstock integrable on $[a, b]$. We write $(H) \int_{I_{0}} f \chi_{E}=(H) \int_{E} f$.

Definition 1.2 (see $[1,5,7]$ ). A function $f:[a, b] \rightarrow \mathbb{R}$ is Denjoy (or special Denjoy) integrable if there exists an $A C G$ (or $A C G^{*}$ ) function $F:[a, b] \rightarrow \mathbb{R}$ such that $D_{\mathrm{ap}} F(t)=$ $f(t)$ (or $F^{\prime}(t)=f(t)$ ) almost everywhere on $[a, b]$. Where $D_{\text {ap }} F(t)$ denotes the approximate derivative of $F$ at $t$. We write $(D) \int_{I_{0}} f=F\left(I_{0}\right)$ (or $\left(D^{*}\right) \int_{I_{0}} f=F\left(I_{0}\right)$ ).

The function $f$ is said to be Denjoy (or special Denjoy) integrable on the set $E \subset$ $[a, b]$ if the function $f \chi_{E}$ is Denjoy (or special Denjoy) integrable on $[a, b]$. We write (D) $\int_{I_{0}} f \chi_{E}=(D) \int_{E} f$ (or $\left.\left(D^{*}\right) \int_{I_{0}} f \chi_{E}=\left(D^{*}\right) \int_{E} f\right)$.

If $f$ is special Denjoy integrable, then $f$ is Denjoy integrable.

LEMmA 1.3 (see [7]). A function $f:[a, b] \rightarrow \mathbb{R}$ is Henstock integrable on $[a, b]$ if and only if $f$ is the special Denjoy integrable on $[a, b]$.

Definition 1.4 (see Gordon [5]). (a) A function $f:[a, b] \rightarrow X$ is Denjoy-Dunford integrable on $[a, b]$ if for each $x^{*}$ in $X^{*}$ the function $x^{*} f$ is Denjoy integrable on $[a, b]$ and if for every interval $I$ in $[a, b]$ there exists a vector $x_{I}^{* *}$ in $X^{* *}$ such that $x_{I}^{* *}\left(x^{*}\right)=\int_{I} x^{*} f$ for all $x^{*}$ in $X^{*}$. We write $x_{I_{0}}^{* *}=(D D) \int_{I_{0}} f=F\left(I_{0}\right)$ and $F$ is called the primitive of $f$ on $I_{0}$.

(b) A function $f:[a, b] \rightarrow X$ is Denjoy-Pettis integrable on $[a, b]$ if $f$ is DenjoyDunford integrable on $[a, b]$ and if $x_{I}^{* *} \in X$ for every interval $I$ in $[a, b]$. We write $x_{I_{0}}^{* *}=(D P) \int_{I_{0}} f=F\left(I_{0}\right)$ and $F$ is called the primitive of $f$ on $I_{0}$.

The function $f$ is said to be integrable in one of the above senses on the set $E \subset[a, b]$ if the function $f \chi_{E}$ is integrable in that sense on $[a, b]$.

LEMma 1.5 (see [3]). A function $f:[a, b] \rightarrow X$ is Denjoy-Dunford integrable on $[a, b]$ if and only if $x^{*} f$ is Denjoy integrable on $[a, b]$ for all $x^{*} \in X^{*}$.

2. Definition and properties. In the following, we give the Riemann-type extensions of Dunford, Pettis integrals, and discuss the relationships between HenstockDunford integral and Dunford integral, Henstock-Pettis integral and Pettis integral.

DEFINITION 2.1. (a) A function $f:[a, b] \rightarrow X$ is Henstock-Dunford integrable on $[a, b]$ if for each $x^{*}$ in $X^{*}$ the function $x^{*} f$ is Henstock integrable on $[a, b]$ and if for every interval $I$ in $[a, b]$ there exists a vector $x_{I}^{* *}$ in $X^{* *}$ such that $x_{I}^{* *}\left(x^{*}\right)=\int_{I} x^{*} f$ for all $x^{*}$ in $X^{*}$. We write $x_{I_{0}}^{* *}=(H D) \int_{I_{0}} f=F\left(I_{0}\right)$ and $F$ is called the primitive of $f$ on $I_{0}$.

(b) A function $f:[a, b] \rightarrow X$ is Henstock-Pettis integrable on $[a, b]$ if $f$ is HenstockDunford integrable on $[a, b]$ and if $x_{I}^{* *} \in X$ for every interval $I$ in $[a, b]$. We write $x_{I_{0}}^{* *}=(H P) \int_{I_{0}} f=F\left(I_{0}\right)$ and $F$ is called the primitive of $f$ on $I_{0}$.

The function $f$ is said to be integrable in one of the above senses on the set $E \subset[a, b]$ if the function $f \chi_{E}$ is integrable in that sense on $[a, b]$.

By the above definitions and Definition 1.4, it is easy to see that if $f$ is HenstockDunford (or Henstock-Pettis) integrable on $I_{0}$, then $f$ is Denjoy-Dunford (or DenjoyPettis) integrable. 
THEOREM 2.2. A function $f:[a, b] \rightarrow X$ is Henstock-Dunford integrable on $[a, b]$ if and only if $x^{*} f$ is Henstock integrable on $[a, b]$ for all $x^{*} \in X^{*}$.

Proof. If $f$ is Henstock-Dunford integrable on $[a, b]$, for every $x^{*} \in X^{*}$, by Definition 2.1, $x^{*} f$ is Henstock integrable on $[a, b]$. Conversely, if $x^{*} f$ is Henstock integrable on $[a, b]$. It follows from Lemma 1.3 that $x^{*} f$ is Denjoy integrable on $[a, b]$ and $(D) \int_{a}^{b} x^{*} f=(H) \int_{a}^{b} x^{*} f$. It follows from Lemma 1.5 that $f$ is Denjoy-Dunford integrable on $[a, b]$, and for every interval $I$ in $[a, b]$ there exists a vector $x_{I}^{* *}$ in $X^{* *}$ such that $x_{I}^{* *}\left(x^{*}\right)=(D) \int_{I} x^{*} f$ for all $x^{*}$ in $X^{*}$, that is, $x_{I}^{* *}\left(x^{*}\right)=(H) \int_{I} x^{*} f$ for all $x^{*}$ in $X^{*}$. So $f$ is Henstock-Dunford integrable on $[a, b]$.

THEOREM 2.3. If the function $f:[a, b] \rightarrow X$ is Henstock-Dunford integrable on $[a, b]$, then each perfect set in $[a, b]$ contains a portion on which $f$ is Dunford integrable.

Proof. Since the function $f:[a, b] \rightarrow X$ is Henstock-Dunford integrable on $[a, b]$, then for each $x^{*} \in X^{*}, x^{*} f$ is Henstock integrable on $[a, b]$. It follows from [8] that each perfect set in $[a, b]$ contains a portion on which $x^{*} f$ is Lebesgue integrable. So $f$ is Dunford integrable on a portion.

THEOREM 2.4. If the function $f:[a, b] \rightarrow X$ is Henstock-Dunford integrable on $[a, b]$, then there is a sequence $\left\{X_{k}\right\}$ of closed subsets such that $X_{k} \subset X_{k+1}$ for all $k, \cup_{k=1}^{\infty} X_{K}=$ $[a, b], f$ is Dunford integrable on each $X_{k}$ and

$$
\lim _{k \rightarrow \infty}(\text { Dunford }) \int_{X_{k} \cap[a, x]} f(t) d t=(H D) \int_{a}^{x} f(t) d t \text { weakly }
$$

uniformly on $[a, b]$.

Proof. It follows from Theorem 2.2 that a function $f:[a, b] \rightarrow X$ is HenstockDunford integrable on $[a, b]$ if and only if $x^{*} f$ is Henstock integrable on $[a, b]$ for all $x^{*} \in X^{*}$. From [8], $x^{*} f$ is Henstock integrable on $[a, b]$, then there is a sequence $\left\{X_{k}\right\}$ of closed subsets such that $X_{k} \subset X_{k+1}$ for all $k, \cup_{k=1}^{\infty} X_{K}=[a, b], x^{*} f$ is Lebesgue integrable on each $X_{k}$ and

$$
\lim _{k \rightarrow \infty}(L) \int_{X_{k} \cap[a, x]} x^{*} f(t) d t=(H) \int_{a}^{x} x^{*} f(t) d t
$$

uniformly on $[a, b]$ for each $x^{*} \in X^{*}$. So we obtain that $f$ is Dunford integrable on each $X_{k}$ and

$$
\lim _{k \rightarrow \infty} \text { (Dunford) } \int_{X_{k} \cap[a, x]} f(t) d t=(H D) \int_{a}^{x} f(t) d t \text { weakly }
$$

uniformly on $[a, b]$.

THEOREM 2.5. If the function $f:[a, b] \rightarrow X$ is Henstock-Dunford integrable on $[a, b]$, then there exists a sequence $\left\{X_{k}\right\}$ of closed sets, $\cup_{k=1}^{\infty} X_{k}=[a, b], f$ is Dunford integrable on each $X_{k}$.

Proof. Since $f$ Henstock-Dunford integrable on $[a, b]$, by Definition 2.1, for each $x^{*} \in X^{*}, x^{*} f$ is Henstock integrable on $[a, b]$, and for every interval $I \subset[a, b]$, 
$\int_{I} x^{*} f=x^{*} \int_{I} f$, and $F(I)=\int_{I} f \in X$. Since $x^{*} f$ is Henstock integrable, then $x^{*} F$ is $A C G^{*}$. So there is a sequence $\left\{X_{k}\right\}$ of closed subsets such that $\cup_{k=1}^{\infty} X_{k}=[a, b]$ and $x^{*} F$ is $V B^{*}$ on each $X_{k}$. From [7, Lemma 6.18], $x^{*} f$ is Lebesgue integrable on each $X_{k}$. So we obtain that $f$ is Dunford integrable on each $X_{k}$.

THEOREM 2.6. Suppose that $X$ contains no copy of $c_{0}$ and $f:[a, b] \rightarrow X$. If the function $f$ is Henstock-Pettis integrable on $[a, b]$, then each perfect set in $[a, b]$ contains a portion on which $f$ is Pettis integrable.

Proof. Since the function $f:[a, b] \rightarrow X$ is Henstock-Pettis integrable on $[a, b]$, then $f$ is Denjoy-Pettis integrable on $[a, b]$. It follows from [5, Theorem 38] that each perfect set in $[a, b]$ contains a portion on which $f$ is Pettis integrable.

In the fact, from [3, Theorem 10], we have that if each Henstock-Pettis integrable function defined on $[a, b]$ is Pettis integrable on a portion of every close set, then $X$ does not contain $c_{0}$.

THEOREM 2.7. Suppose that $X$ contains no copy of $c_{0}$ and $f:[a, b] \rightarrow X$ is a measurable. If the function $f:[a, b] \rightarrow X$ is Henstock-Pettis integrable on $[a, b]$, then there exists a sequence $\left\{X_{k}\right\}$ of closed sets with $X_{k} \uparrow[a, b]$ such that for each $x^{*} \in X^{*}, f$ is Pettis integrable on each $X_{k}$, and

$$
\lim _{k \rightarrow \infty}(\text { Pettis }) \int_{X_{k}} f=(H P) \int_{a}^{b} f \text { weakly. }
$$

Proof. Since $f$ is Henstock-Pettis integrable on $[a, b]$, then $f$ is Henstock-Dunford integrable on $[a, b]$. By Theorem 2.4, there is a sequence $\left\{X_{k}\right\}$ of closed subsets such that $X_{k} \subset X_{k+1}$ for all $k, \cup_{k=1}^{\infty} X_{K}=[a, b], x^{*} f$ is Lebesgue integrable on each $X_{k}$ and

$$
\lim _{k \rightarrow \infty}(L) \int_{X_{k} \cap[a, x]} x^{*} f(t) d t=(H) \int_{a}^{x} x^{*} f(t) d t
$$

uniformly on $[a, b]$ for each $x^{*} \in X^{*}$. So we obtain that $f$ is Dunford integrable on each $X_{k}$. From [2, Theorem 2.5, page 54], $f$ is Pettis integrable on $[a, b]$ and

$$
\lim _{k \rightarrow \infty}(\text { Pettis }) \int_{X_{k} \cap[a, x]} f(t) d t=(H P) \int_{a}^{x} f(t) d t \text { weakly }
$$

uniformly on each $X_{k}$, that is,

$$
\lim _{k \rightarrow \infty} \text { (Pettis) } \int_{X_{k}} f=(H P) \int_{a}^{b} f \text { weakly. }
$$

In Theorem 2.7, if we remove the condition that $f$ is a measurable, then we have the following theorem.

THEOREM 2.8. Suppose that $X$ contains no copy of $c_{0}$. If the function $f:[a, b] \rightarrow X$ is Henstock-Pettis integrable on $[a, b]$, then there exists a sequence $\left\{X_{k}\right\}$ of closed sets, $\cup_{k=1}^{\infty} X_{k}=[a, b], f$ is Pettis integrable on each $X_{k}$.

Proof. Since $f$ is Henstock-Pettis integrable on $[a, b]$, by Definition 2.1, for each $x^{*} \in X^{*}, x^{*} f$ is Henstock integrable on $[a, b]$, and for every interval $I \subset[a, b]$, 
$\int_{I} x^{*} f=x^{*} \int_{I} f$, and $F(I)=\int_{I} f \in X$. Since $x^{*} f$ is Henstock integrable, then $x^{*} F$ is $A C G^{*}$. So there is a sequence $\left\{X_{k}\right\}$ of closed subsets such that $\cup_{k=1}^{\infty} X_{k}=[a, b]$ and $x^{*} F$ is $V B^{*}$ on each $X_{k}$. For each $k \in N$, let $(a, b)-X_{k}=\cup_{n=1}^{\infty}\left(c_{n}^{k}, d_{n}^{k}\right)$. Then

$$
\sum_{n=1}^{\infty}\left|x^{*} \int_{c_{n}^{k}}^{d_{n}^{k}} f\right|<\infty
$$

Since $X$ contains no copy of $c_{0}$, by Bessaga-Pelczynski theorem [2, page 22], $\sum_{n=1}^{\infty} \int_{c_{n}^{k}}^{d_{n}^{k}} f$ is unconditionally convergent in norm. Also

$$
\sum_{n=1}^{\infty} \sup _{\left[a_{n}^{k}, b_{n}^{k}\right] \subset\left[c_{n}^{k}, d_{n}^{k}\right]}\left|x^{*} \int_{a_{n}^{k}}^{b_{n}^{k}} f\right|<\infty .
$$

By Harnack extension theorem [7, page 41], we have

$$
\int_{X_{k}} x^{*} f=\int_{a}^{b} x^{*} f-\sum_{n=1}^{\infty} \int_{c_{n}^{k}}^{d_{n}^{k}} x^{*} f=x^{*}\left(\int_{a}^{b} f-\sum_{n=1}^{\infty} \int_{c_{n}^{k}}^{d_{n}^{k}} f\right) .
$$

Hence $\int_{X_{k}} f=\int_{a}^{b} f-\sum_{n=1}^{\infty} \int_{c_{n}^{k}}^{d_{n}^{k}} f \in X$ and $\int_{X_{k}} x^{*} f=x^{*} \int_{X_{k}} f$.

So, for every closed set $H \subset X_{k}$, we have $\int_{H} x^{*} f=x^{*} \int_{H} f$ and $\int_{H} f \in X$. Since $\int_{a}^{b} f X_{X_{k}}=\int_{X_{k}} f \in X, \int_{a}^{b} f \chi_{H}=\int_{H} f \in X$, then for every closed interval $I \subset[a, b]$, $\int_{I} f X_{X_{k}}=\int_{I \cap X_{k}} f \in X$. By [5, Theorem 23, page 79], $f X_{X_{k}}$ is Pettis integrable on $[a, b]$, that is, $f$ is Pettis integrable on each $X_{k}$.

3. The extension theorems and convergence theorems. Now we consider the extension theorems and convergence theorems of the Henstock-Dunford and HenstockPettis integrals.

THEOREM 3.1. Let $E$ be a closed subset in $[a, b]$ and $(a, b)-E$ the union of $\left\{\left(a_{k}, b_{k}\right)\right\}$, $k=1,2, \ldots$ If $f:[a, b] \rightarrow X$ is Henstock-Dunford integrable on $E$ and each interval $\left[a_{k}, b_{k}\right]$ with

$$
\sum_{k=1}^{\infty} \omega\left(\int_{a_{k}}^{t} x^{*} f,\left[a_{k}, b_{k}\right]\right)<\infty
$$

for each $x^{*} \in X^{*}$, then $f$ is Henstock-Dunford integrable on $[a, b]$ and

$$
\left\langle x^{*},(H D) \int_{a}^{b} f\right\rangle=\left\langle x^{*},(H D) \int_{a}^{b} f \chi_{E}\right\rangle+\sum_{k=1}^{\infty}\left\langle x^{*},(H D) \int_{a_{k}}^{b_{k}} f\right\rangle
$$

for each $x^{*} \in X^{*}$.

Proof. From the conditions of Theorem 3.1, we have the function $x^{*} f$ satisfies the hypothesis of [7, Corollary 7.11]. So we have $x^{*} f$ is Henstock integrable on $[a, b]$ and

$$
(H) \int_{a}^{b} x^{*} f=(H) \int_{a}^{b} x^{*} f \chi_{E}+\sum_{k=1}^{\infty}(H) \int_{a_{k}}^{b_{k}} x^{*} f .
$$


It follows from Theorem 2.2 that $f$ is Henstock-Dunford integrable on $[a, b]$ and the above equality means that

$$
\left\langle x^{*},(H D) \int_{a}^{b} f\right\rangle=\left\langle x^{*},(H D) \int_{a}^{b} f \chi_{E}\right\rangle+\sum_{k=1}^{\infty}\left\langle x^{*},(H D) \int_{a_{k}}^{b_{k}} f\right\rangle
$$

for each $x^{*} \in X^{*}$.

THEOREM 3.2. Let $E$ be a closed subset in $[a, b]$ and $\left\{\left(a_{k}, b_{k}\right)\right\}$ be an enumeration of the intervals contiguous to $E$ in $(a, b)$. Suppose that $f:[a, b] \rightarrow X$ is Henstock-Pettis integrable on $E$ and each interval $\left[a_{k}, b_{k}\right]$. If $\sum_{k=1}^{\infty} \omega\left(\int_{a_{k}}^{t} x^{*} f,\left[a_{k}, b_{k}\right]\right)<\infty$ for each $x^{*} \in X^{*}$ and the series $\sum_{k=1}^{\infty}(H P) \int_{\left[a_{k}, b_{k}\right] \cap J} f$ is unconditionally convergent for every subinterval $J$ of $[a, b]$, then $f$ is Henstock-Pettis integrable on $[a, b]$ and

$$
(H P) \int_{a}^{b} f=(H P) \int_{a}^{b} f \chi_{E}+\sum_{k=1}^{\infty}(H P) \int_{a_{k}}^{b_{k}} f .
$$

Proof. From Theorem 3.1, we have the function $f$ is Henstock-Dunford integrable on $[a, b]$ and $(H) \int_{a}^{b} x^{*} f=(H) \int_{a}^{b} x^{*} f \chi_{E}+\sum_{k=1}^{\infty}(H) \int_{a_{k}}^{b_{k}} x^{*} f$. To show that $f$ is in fact Henstock-Pettis integrable on $[a, b]$. We need to show that $(H D) \int_{J} f$ belongs to $X$ for each closed interval $J$ in $[a, b]$.

Let $E_{0}=E \cap J$. Then $E_{0}$ is a closed set. Since $f \chi_{E}$ is Henstock-Pettis integrable on $J$, then $f \chi_{E_{0}}$ is Henstock-Pettis integrable on $J$, that is, $f$ is Henstock-Pettis integrable on $E_{0}$. And $\left\{\left(a_{k}, b_{k}\right) \cap J\right\}$ is an enumeration of the intervals contiguous to $E_{0}$ in $J$, so $f$ is Henstock-Pettis integrable on them and $\sum_{k}(H P) \int_{\left[a_{k}, b_{k}\right] \cap J} f$ is an unconditionally convergent series in $X$. Now, if we apply Theorem 3.1 to $E_{0}$ in $J$, we get

$$
\left\langle x^{*},(H D) \int_{J} f\right\rangle=\left\langle x^{*},(H P) \int_{J} f \chi_{E_{0}}\right\rangle+\sum_{k=1}^{\infty}\left\langle x^{*},(H P) \int_{\left[a_{k}, b_{k}\right] \cap J} f\right\rangle
$$

for each $x^{*} \in X^{*}$, that is,

$$
\left\langle x^{*},(H D) \int_{J} f\right\rangle=\left\langle x^{*},(H P) \int_{J} f \chi_{E_{0}}+\sum_{k=1}^{\infty}(H P) \int_{\left[a_{k}, b_{k}\right] \cap J} f\right\rangle
$$

for each $x^{*} \in X^{*}$. We conclude that

$$
(H D) \int_{J} f=(H P) \int_{J} f \chi_{E_{0}}+\sum_{k=1}^{\infty}(H P) \int_{\left[a_{k}, b_{k}\right] \cap J} f .
$$

Hence, $f$ is Henstock-Pettis integrable on $[a, b]$ and

$$
(H P) \int_{a}^{b} f=(H P) \int_{a}^{b} f \chi_{E_{0}}+\sum_{k=1}^{\infty}(H P) \int_{\left[a_{k}, b_{k}\right] \cap J} f .
$$

COROLLARY 3.3. Suppose that $X$ contains no copy of $c_{0}$. Let $E$ be a closed subset in $[a, b]$ and $\left\{\left(a_{k}, b_{k}\right)\right\}$ be an enumeration of the intervals contiguous to $E$ in $(a, b)$. Suppose that $f:[a, b] \rightarrow X$ is Henstock-Pettis integrable on $E$ and each interval $\left[a_{k}, b_{k}\right]$. 
If $\sum_{k=1}^{\infty} \omega\left(\int_{a_{k}}^{t} x^{*} f,\left[a_{k}, b_{k}\right]\right)<\infty$ for each $x^{*} \in X^{*}$, then $f$ is Henstock-Pettis integrable on $[a, b]$ and

$$
(H P) \int_{a}^{b} f=(H P) \int_{a}^{b} f \chi_{E}+\sum_{k=1}^{\infty}(H P) \int_{a_{k}}^{b_{k}} f
$$

THEOREM 3.4. Suppose that $X$ is weakly sequentially complete and $f:[a, b] \rightarrow X$ is Henstock-Dunford integrable on $[a, b]$. If $f$ is measurable, then $f$ is Henstock-Pettis integrable on $[a, b]$.

Proof. It is similar to the proof of [5, Theorem 40].

LEMMA 3.5 (see $[1,5])$. Let $\Gamma$ be a family of open intervals in $(a, b)$ and suppose that $\Gamma$ has the following properties:

(1) if $(\alpha, \beta)$ and $(\beta, \gamma)$ belong to $\Gamma$, then $(\alpha, \gamma)$ belongs to $\Gamma$;

(2) if $(\alpha, \beta)$ belong to $\Gamma$, then every open interval in $(\alpha, \beta)$ belongs to $\Gamma$;

(3) if $(\alpha, \beta)$ belong to $\Gamma$ for every interval in $[\alpha, \beta] \subset(c, d)$, then $(c, d)$ belongs to $\Gamma$;

(4) if all of the intervals contiguous to the perfect set $E \subset[a, b]$ belong to $\Gamma$, then there exists an interval $I$ in $\Gamma$ such that $I \cap E \neq \varnothing$.

Then $\Gamma$ contains the interval $(a, b)$.

LEMMA 3.6. Suppose that $f_{n}:[a, b] \rightarrow \mathbb{R}, f:[a, b] \rightarrow \mathbb{R}$, and

(1) $f_{n} \rightarrow f$ almost everywhere on $[a, b]$ as $n \rightarrow \infty$, where each $f_{n}$ is Henstock (or $\left.D^{*}\right)$ integrable on $[a, b]$;

(2) the primitives $F_{n}$ of $f_{n}$ are continuous uniformly in $n$ and $A C G^{*}$ uniformly in $n$. Then $f$ is Henstock (or $D^{*}$ ) integrable on $[a, b]$ and

$$
\lim _{n \rightarrow \infty} \int_{a}^{b} f_{n}=\int_{a}^{b} f
$$

DEFINITION 3.7. Let $F:[a, b] \rightarrow X$ and let $E$ be a subset of $[a, b]$.

(a) $F$ is $B V^{*}$ on $E$ if $\sup \left\{\sum_{i} \omega\left(F ;\left[c_{i}, d_{i}\right]\right)\right\}$ is finite, where the supremum is taken over all finite collections $\left\{\left[c_{i}, d_{i}\right]\right\}$ of nonoverlapping intervals that have endpoints in $E$, $\omega$ denotes the oscillation of $F$ over $\left[c_{i}, d_{i}\right]$, that is,

$$
\omega\left(F ;\left[c_{i}, d_{i}\right]\right)=\sup \left\{\|F(x)-F(y)\| ; x, y \in\left[c_{i}, d_{i}\right]\right\} .
$$

(b) $F$ is $A C^{*}$ on $E$ if for each $\epsilon>0$ there exists $\delta>0$ such that $\sum_{i} \omega\left(F ;\left[c_{i}, d_{i}\right]\right)<\epsilon$ whenever $\left\{\left[c_{i}, d_{i}\right]\right\}$ is a finite collection of nonoverlapping intervals that have endpoints in $E$ and satisfy $\sum_{i}\left(d_{i}-c_{i}\right)<\delta$.

(c) $F$ is $B V G^{*}$ on $E$ if $E$ can be expressed as a countable union of sets on each of which $F$ is $B V^{*}$.

(d) $F$ is $A C G^{*}$ on $E$ if $F$ is continuous on $E$ and if $E$ can be expressed as a countable union of sets on each of which $F$ is $A C^{*}$.

THEOREM 3.8. Suppose that $X$ is weakly sequentially complete and

(1) $f_{n} \rightarrow f$ weakly almost everywhere on $[a, b]$ as $n \rightarrow \infty$, where each $f_{n}$ is HenstockPettis integrable on $[a, b]$;

(2) the primitives $F_{n}$ of $f_{n}$ are continuous uniformly in $n$ and $A C G^{*}$ uniformly in $n$. 
Then $f$ is Henstock-Pettis integrable on $[a, b]$ and

$$
\lim _{n \rightarrow \infty} \int_{a}^{b} f_{n}=\int_{a}^{b} f \text { weakly. }
$$

Proof. Let

$\Gamma=\left\{(\alpha, \beta) \subset[a, b]: f\right.$ is Henstock-Pettis integrable on $[\alpha, \beta], \int_{\alpha}^{\beta} f_{n} \rightarrow \int_{\alpha}^{\beta} f$ weakly $\}$.

We must show that $\Gamma$ contains $(a, b)$ and by Lemma 3.5 it is sufficient to verify that $\Gamma$ satisfies Romanovski's four conditions.

Conditions (1) and (2) are easily verified.

Suppose that $(\alpha, \beta)$ belongs to $\Gamma$ for every interval $[\alpha, \beta]$ in $(c, d)$. For each positive integer $n>2 /(d-c)$, define $I_{n}=(c+1 / n, d-1 / n)$ and let $x_{n}=x_{I_{n}}^{* *}$.

Then we have

$$
x_{(c, d)}^{* *}\left(x^{*}\right)=\int_{c}^{d} x^{*} f=\lim _{n \rightarrow \infty} \int_{I_{n}} x^{*} f=\lim _{n \rightarrow \infty} x^{*}\left(x_{n}\right)
$$

for each $x^{*}$ in $X^{*}$. Since $X$ is weakly sequentially complete, the sequence $\left\{x_{n}\right\}$ converges weakly to an element $x_{0}$ of $X$ and we must have $x_{(c, d)}^{* *}=x_{0}$. It follows easily that $(c, d)$ belongs to $\Gamma$ and this verifies condition (3).

Now let $E$ be a perfect set in $[a, b]$ such that each of the intervals in $[a, b]$ contiguous to $E$ belongs to $\Gamma$.

Since $\left\{F_{n}\right\}$ is continuous uniformly in $n$ and $A C G^{*}$ uniformly in $n$, then for each $x^{*} \in X^{*},\left\{x^{*} F_{n}\right\}$ is continuous uniformly in $n$ and $A C G^{*}$ uniformly in $n$, and $x^{*} f_{n} \rightarrow$ $x^{*} f$ almost everywhere in $[a, b]$. It follows from [1] that $x^{*} f$ is special Denjoy integrable on $[a, b]$. So there exists an interval $[u, v]$ with $u, v \in E$ and $E \cap(u, v) \neq \varnothing$ such that $\left\{F_{n}\right\}$ is $A C^{*}$ uniformly in $n$ on $P=E \cap(u, v)$ and the series $\sum_{k} \omega\left(F_{n} ;\left[u_{k}, v_{k}\right]\right)$ unconditionally converges where $(u, v)-E=\cup_{k}\left(u_{k}, v_{k}\right)$. Hence $\sum_{k} \omega\left(\int_{u_{k}}^{t} x^{*} f_{n} ;\left[u_{k}, v_{k}\right]\right)$ $<\infty$ for each $x^{*} \in X^{*}$. By Corollary 3.3, we have

$$
\int_{u}^{v} f_{n}=\int_{P} f_{n}+\sum_{k} \int_{u_{k}}^{v_{k}} f_{n}
$$

$\left\{F_{n}\right\}$ is $A C^{*}$ uniformly in $n$ on $P,\left\{x^{*} F_{n}: x^{*} \in B\left(X^{*}\right), n \in \mathbb{N}\right\}$ is $A C^{*}$ uniformly in $n$ on $P$. So $\left\{x^{*} f_{n}: x^{*} \in B\left(X^{*}\right), n \in \mathbb{N}\right\}$ is uniformly integrable on $P$ (see [2]), that is, for $E \subset P$,

$$
\lim _{|E| \rightarrow 0} \int_{E}\left|x^{*} f_{n}\right|=0 \quad \text { uniformly in } x^{*} \in B\left(X^{*}\right) \text { and } n .
$$

It follows from [4, Theorem 3] that $f$ is Pettis integrable on $P$ and $\int_{P} f_{n} \rightarrow \int_{P} f$ weakly. Since $\left\{F_{n}\right\}$ is $A C^{*}$ uniformly in $n$ on $P$, so for every $\epsilon>0$ there exists $N$ such that $\sum_{k=N}^{\infty}\left\|\int_{u_{k}}^{v_{k}} f_{n}\right\|<\epsilon, n=1,2, \ldots$ For every $x^{*} \in B\left(X^{*}\right)$, we have $\sum_{k=N}^{\infty}\left|\int_{u_{k}}^{v_{k}} x^{*} f_{n}\right|<\epsilon$, $n=1,2, \ldots$. So $\sum_{k=N}^{\infty}\left|\int_{u_{k}}^{v_{k}} x^{*} f\right|<\epsilon$. Since $X$ is weakly sequentially complete and $X$ does not contain $c_{0}$, hence $\sum_{k} \int_{u_{k}}^{v_{k}} f$ unconditionally converges. By (3.16),

$$
x^{*} \int_{u}^{v} f_{n}=x^{*} \int_{P} f_{n}+x^{*} \sum_{k} \int_{u_{k}}^{v_{k}} f_{n} .
$$


Let $n \rightarrow \infty$, we have

$$
x_{(u, v)}^{* *}\left(x^{*}\right)=x^{*} \int_{P} f+x^{*} \sum_{k} \int_{u_{k}}^{v_{k}} f .
$$

Hence

$$
x_{(u, v)}^{* *}=\int_{P} f+\sum_{k} \int_{u_{k}}^{v_{k}} f \in X,
$$

that is, $f$ is Henstock-Pettis integrable on $[u, v]$. So $(u, v) \in \Gamma$. This shows that $(u, v)$ belongs to $\Gamma$ and $\Gamma$ satisfies condition (4). This completes the proof.

THEOREM 3.9. Suppose that $X$ is weakly sequentially complete and $f_{n} \rightarrow f$ weakly almost everywhere on $[a, b]$ as $n \rightarrow \infty$, where each $f_{n}$ is Henstock-Pettis integrable on $[a, b]$. If there is a scalar function $g$ with $\left\|f_{n}(\cdot)\right\| \leq g(\cdot)$ almost everywhere for all $n$ and if $\int g<\infty$, then $f$ is Henstock-Pettis integrable on $[a, b]$ and

$$
\lim _{n \rightarrow \infty} \int_{a}^{b} f_{n}=\int_{a}^{b} f \text { weakly. }
$$

Proof. It is similar to the proof of Theorem 3.8.

DEFINITION 3.10. Let $\left\{f_{\alpha}\right\}$ be a family of Henstock-Pettis integrable functions defined on $[a, b]$. The family $\left\{x^{*} f_{\alpha}: x^{*} \in B\left(X^{*}\right)\right\}$ is uniformly integrable in the generalized sense on $[a, b]$, if for each perfect set $E \subset[a, b]$ there exists an interval $[c, d] \subset[a, b]$ with $c, d \in E$ and $E \cap(c, d) \neq \varnothing$ such that $\left\{x^{*} f_{\alpha}: x^{*} \in B\left(X^{*}\right)\right\}$ is uniformly integrable on $P=E \cap(c, d)$ and for every $\alpha$ the series $\sum_{k} \int_{\mathcal{C}_{k}}^{d_{k}} f_{\alpha}$ is unconditionally convergent where $(c, d)-E=\cup_{k}\left(c_{k}, d_{k}\right)$.

THEOREM 3.11. Suppose that $X$ is weakly sequentially complete and

(1) $f_{n} \rightarrow f$ weakly almost everywhere on $[a, b]$ as $n \rightarrow \infty$, where each $f_{n}$ is HenstockPettis integrable on $[a, b]$.

(2) The family $\left\{x^{*} f_{n}: x^{*} \in B\left(X^{*}\right), n \in \mathbb{N}\right\}$ is uniformly integrable in the generalized sense on $[a, b]$.

(3) For each $x^{*} \in X^{*}, \lim _{n \rightarrow \infty} \int_{c}^{d} x^{*} f_{n}=\int_{c}^{d} x^{*} f$ uniformly for every $[c, d] \subset[a, b]$.

Then $f$ is Henstock-Pettis integrable on $[a, b]$ and

$$
\lim _{n \rightarrow \infty} \int_{a}^{b} f_{n}=\int_{a}^{b} f \text { weakly. }
$$

Proof. It is similar to the proof of Theorem 3.8. The only difference is that the family $\left\{x^{*} f_{n}: x^{*} \in B\left(X^{*}\right), n \in \mathbb{N}\right\}$ is uniformly integrable in the generalized sense on $[a, b]$, then there is a portion $P=E \cap I$ of $E$ such that the family $\left|x^{*} f_{n} X_{E}\right|$ is uniformly integrable on $P$. So $f$ is Pettis integrable on $P$.

THEOREM 3.12. Suppose that $X$ is weakly sequentially complete and

(1) $f_{n} \rightarrow f$ weakly almost everywhere on $[a, b]$ as $n \rightarrow \infty$, where each $f_{n}$ is HenstockPettis integrable on $[a, b]$ and $f$ is measurable,

(2) the primitives $F_{n}$ of $f_{n}$ are weakly continuous uniformly in $n$ and weakly ACG* uniformly in $n$, that is, for every $x^{*} \in X^{*}, x^{*} F_{n}$ are continuous uniformly in $n$ and $A C G^{*}$ uniformly in $n$. 
Then $f$ is Henstock-Pettis integrable on $[a, b]$ and

$$
\lim _{n \rightarrow \infty} \int_{a}^{b} f_{n}=\int_{a}^{b} f \text { weakly. }
$$

Proof. For each $x^{*}$ in $X^{*}$, we have

(1) $x^{*} f_{n} \rightarrow x^{*} f$ almost everywhere on $[a, b]$ as $n \rightarrow \infty$, where each $x^{*} f_{n}$ is Henstock integrable on $[a, b]$,

(2) the primitives $x^{*} F_{n}$ of $x^{*} f_{n}$ are continuous uniformly in $n$ and $A C G^{*}$ uniformly in $n$. It follows from Lemma 3.6 that $x^{*} f$ is Henstock integrable on $[a, b]$ and

$$
\int_{a}^{b} x^{*} f_{n} \rightarrow \int_{a}^{b} x^{*} f \quad \text { as } n \rightarrow \infty .
$$

By Theorem 2.2, $f$ is Henstock-Dunford integrable on $[a, b]$. Since $X$ is weakly sequentially complete and $f$ is measurable, by Theorem 3.4, $f$ is Henstock-Pettis integrable on $[a, b]$.

THEOREM 3.13. Suppose that the unit ball $B\left(X^{*}\right)$ of $X^{*}$ is weak* sequentially compact and

(1) $f_{n} \rightarrow f$ weakly almost everywhere in $[a, b]$ as $n \rightarrow \infty$, where each $f_{n}$ is HenstockPettis integrable on $[a, b]$,

(2) the primitives $F_{n}$ of $f_{n}$ are continuous uniformly in $n$ and $A C G^{*}$ uniformly in $n$.

Then $f$ is Henstock-Pettis integrable on $[a, b]$ and

$$
\lim _{n \rightarrow \infty} \int_{a}^{b} f_{n}=\int_{a}^{b} f \text { weakly. }
$$

Proof. Suppose that $I \subset I_{0}$. Let $C$ be the weak closure of $\left\{\int_{I} f_{n}: n \in \mathbb{N}\right\}$. For each $x^{*}$ in $X^{*},\left\{x^{*} F_{n}: n \in \mathbb{N}\right\}$ is continuous uniformly in $n$ and $A C G^{*}$ uniformly in $n$ in $[a, b]$, and further $\int_{a}^{b} x^{*} f_{n}=x^{*} \int_{a}^{b} f_{n}$. A convergence theorem, namely Lemma 3.6, guarantees that $x^{*} f$ is Henstock integrable on $[a, b]$ and $\lim _{n \rightarrow \infty} \int_{a}^{b} x^{*} f_{n}=\int_{a}^{b} x^{*} f$ for each $x^{*}$ in $X^{*}$. We observe that $C$ is bounded and that $C-\left\{\int_{I} f_{n}: n \in \mathbb{N}\right\}$ contains at most one point. We will prove that $C$ is weakly compact.

Suppose that $C$ is not weakly compact. An appeal to a theorem of James [6, Theorem 1] produces a bounded sequence $\left(x_{k}^{*}\right)$ in $X^{*}$, a sequence $\left(x_{n}\right)$ in $C$, and an $\epsilon>0$ such that $x_{k}^{*}\left(x_{n}\right)=0$ for $k>n$ and $x_{k}^{*}\left(x_{n}\right)>\epsilon$ for $n \geq k$. By passing to subsequences and relabelling, we can find a subsequence $\left(\int_{I} g_{n}\right)$ of $\left(\int_{I} f_{n}\right)$ and a subsequence $\left(y_{k}^{*}\right)$ of $x_{k}^{*}$ such that

$$
\begin{array}{ll}
y_{k}^{*} \int_{I} g_{n}=\int_{I} y_{k}^{*} g_{n}=0 & \text { for } k>n, \\
y_{k}^{*} \int_{I} g_{n}=\int_{I} y_{k}^{*} g_{n}>\epsilon & \text { for } n \geq k, \\
\lim _{n \rightarrow \infty} \int_{I} x^{*} g_{n}=\int_{I} x^{*} f \quad \forall x^{*} \text { in } X^{*} .
\end{array}
$$

Since the unit ball $B\left(X^{*}\right)$ of $X^{*}$ is weak* sequentially compact, the sequence $\left(y_{k}^{*}\right)$ has a subsequence $\left(y_{k_{j}}^{*}\right)$ which weak* converges to $y_{0}^{*}$, so $\lim _{j \rightarrow \infty} y_{k_{j}}^{*} f=y_{0}^{*} f$ on $I_{0}$, 
$\lim _{j \rightarrow \infty} y_{k_{j}}^{*} F=y_{0}^{*} F$ on $I_{0}$, that is, $\lim _{j \rightarrow \infty} \int_{I} y_{k_{j}}^{*} f=\int_{I} y_{0}^{*} f$. To force a contradiction, note that for each $k, \lim _{n \rightarrow \infty} \int_{I} y_{k}^{*} f_{n}=\int_{I} y_{k}^{*} f$. Hence $\int_{I} y_{k}^{*} f \geq \epsilon$ for each $\mathrm{k}$, and $\int_{I} y_{0}^{*} f \geq \epsilon$. On the other hand, notice that since each $g_{n}$ is Henstock-Pettis integrable, $\left(y_{k_{j}}^{*}\right)$ weak* converges to $y_{0}^{*}$, hence

$$
\lim _{j \rightarrow \infty} \int_{I} y_{k_{j}}^{*} g_{n}=\lim _{j \rightarrow \infty} y_{k_{j}}^{*} \int_{I} g_{n}=y_{0}^{*} \int_{I} g_{n}=\int_{I} y_{0}^{*} g_{n} .
$$

Since this holds for each $n$, and since $\lim _{n \rightarrow \infty} \int_{I} y_{0}^{*} g_{n}=\int_{I} y_{0}^{*} f$, we see that $\int_{I} y_{0}^{*} f=0$. This contradicts the inequality $\int_{I} y_{0}^{*} f \geq \epsilon$, and proves that $C$ is weakly compact. Since $\lim _{n \rightarrow \infty} \int_{I} x^{*} f_{n}=\int_{I} x^{*} f$, the sequence $\left(\int_{I} f_{n}\right)$ of the Henstock-Pettis integrals is weakly Cauchy. It follows from the weak compactness of $C$ that $\lim _{n \rightarrow \infty} \int_{I} f_{n}$ exists weakly in $X$. Denote $F(I)=\int_{I} f=\lim _{n \rightarrow \infty} \int_{I} f_{n}$ weakly, then $x^{*} F(I)=x^{*} \int_{I} f=\int_{I} x^{*} f$ for each $x^{*}$ in $X^{*}$. So $f$ is Henstock-Pettis integrable on $[a, b]$ and

$$
\lim _{n \rightarrow \infty} \int_{a}^{b} f_{n}=\int_{a}^{b} f \text { weakly. }
$$

COROLLARY 3.14. Suppose that $X$ is a reflexive Banach space and

(1) $f_{n} \rightarrow f$ weakly almost everywhere on $[a, b]$ as $n \rightarrow \infty$, where each $f_{n}$ is HenstockPettis integrable on $[a, b]$,

(2) the primitives $F_{n}$ of $f_{n}$ are weakly continuous uniformly in $n$ and weakly ACG* uniformly in $n$ on $[a, b]$.

Then $f$ is Henstock-Pettis integrable on $[a, b]$ and

$$
\lim _{n \rightarrow \infty} \int_{a}^{b} f_{n}=\int_{a}^{b} f \text { weakly. }
$$

THEOREM 3.15. If the following conditions are satisfied:

(1) $\lim _{n \rightarrow \infty} f_{n}=f$ weakly almost everywhere on $[a, b]$, where each $f_{n}$ is HenstockDunford integrable on $[a, b]$,

(2) the primitives $F_{n}$ of $f_{n}$ are weakly continuous uniformly in $n$ and weakly ACG* uniformly in $n$.

Then $f$ is Henstock-Dunford integrable on $[a, b]$ and

$$
\lim _{n \rightarrow \infty} \int_{a}^{b} f_{n}=\int_{a}^{b} f \text { weakly. }
$$

Proof. Since

(1) $\lim _{n \rightarrow \infty} x^{*} f_{n}=x^{*} f$ almost everywhere on $[a, b]$,

(2) the primitives $x^{*} F_{n}$ of $x^{*} f_{n}$ are continuous uniformly in $n$ and $A C G^{*}$ uniformly in $n$.

Then, as in the proof of Theorem 3.12, $x^{*} f$ is Henstock integrable on $[a, b]$ and

$$
\lim _{n \rightarrow \infty} \int_{a}^{b} x^{*} f_{n}=\int_{a}^{b} x^{*} f
$$

By Theorem 2.2, $f$ is Henstock-Dunford integrable on $[a, b]$ and

$$
\lim _{n \rightarrow \infty} \int_{a}^{b} f_{n}=\int_{a}^{b} f \text { weakly. }
$$




\section{REFERENCES}

[1] V. G. Čelidze and A. G. Džvaršeišvili, The Theory of the Denjoy Integral and some Applications, Series in Real Analysis, vol. 3, World Scientific Publishing Co., New Jersey, 1989, translated from Russian, with a preface and an appendix by P. S. Bullen. MR 90k:26013. Zbl 744.26006.

[2] J. Diestel and J. J. Uhl, Jr., Vector Measures, Mathematical Surveys, no. 15, American Mathematical Society, Rhode Island, 1977, with a foreword by B. J. Pettis. MR 56\#12216. Zbl 369.46039.

[3] J. L. Gámez and J. Mendoza, On Denjoy-Dunford and Denjoy-Pettis integrals, Studia Math. 130 (1998), no. 2, 115-133. MR 99k:28013. Zbl 980.39999.

[4] R. F. Geitz, Pettis integration, Proc. Amer. Math. Soc. 82 (1981), no. 1, 81-86. MR 82c:28018. Zbl 506.28007.

[5] R. A. Gordon, The Denjoy extension of the Bochner, Pettis, and Dunford integrals, Studia Math. 92 (1989), no. 1, 73-91. MR 90b:28011. Zbl 681.28006.

[6] R. C. James, Weak compactness and reflexivity, Israel J. Math. 2 (1964), 101-119. MR 31\#585. Zbl 127.32502.

[7] P. Y. Lee, Lanzhou Lectures on Henstock Integration, Series in Real Analysis, vol. 2, World Scientific Publishing Co., New Jersey, 1989. MR 92j:26010. Zbl 699.26004.

[8] G. Q. Liu, On necessary conditions for Henstock integrability, Real Anal. Exchange 18 (1992/93), no. 2, 522-531. MR 94e:26014. Zbl 788.26008.

Ye Guoju: Department of Mathematics, Northwest Normal University, Lanzhou 730070, CHINA

E-mail address: yeguoju@21cn.com

AN TIANQING: DEPARTMENT OF MATHEMATICS, NORTHWEST NORMAL UNIVERSITY, LANZHOU 730070, CHINA 


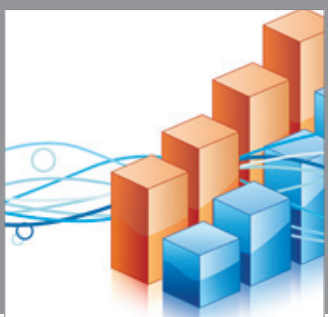

Advances in

Operations Research

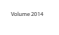

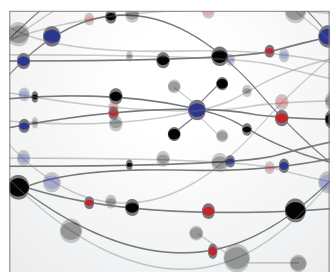

\section{The Scientific} World Journal
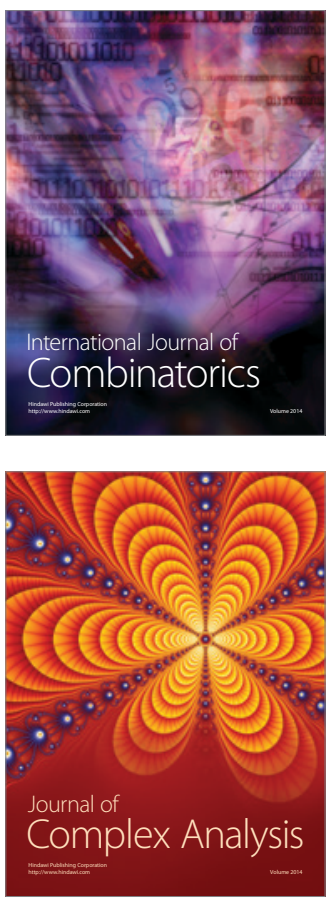

International Journal of

Mathematics and

Mathematical

Sciences
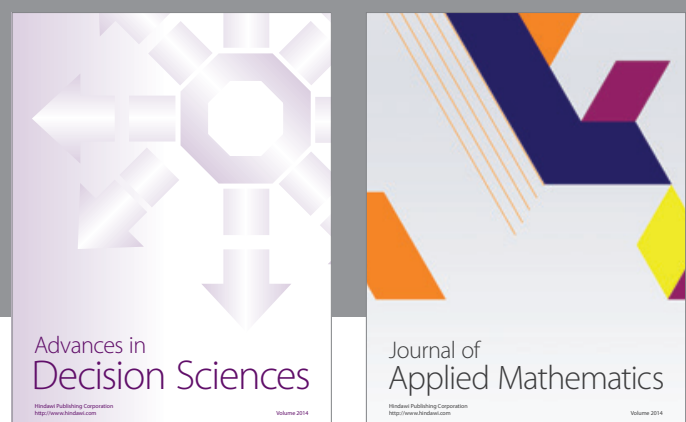

Journal of

Applied Mathematics
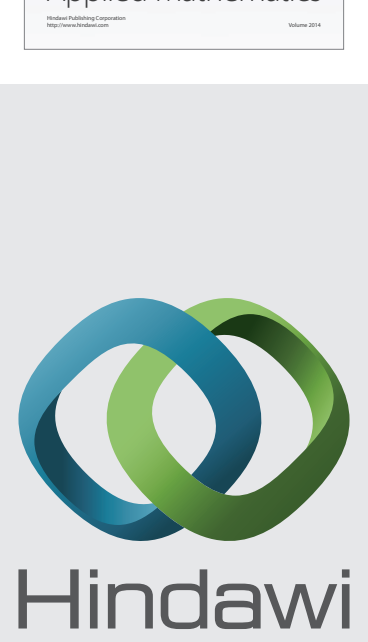

Submit your manuscripts at http://www.hindawi.com
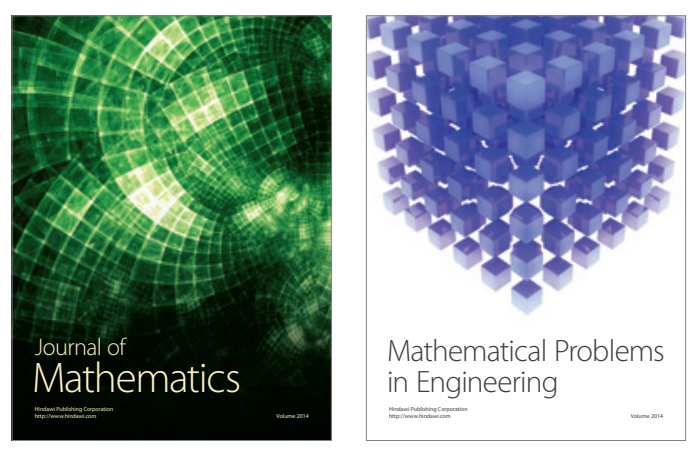

Mathematical Problems in Engineering
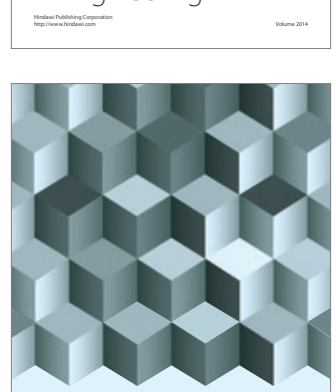

Journal of

Function Spaces
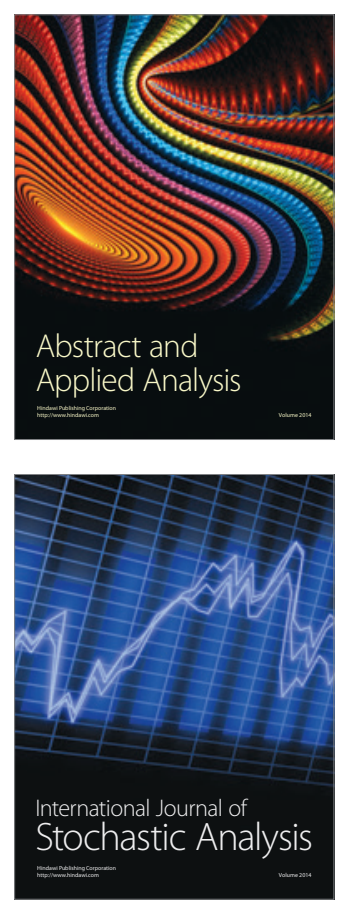

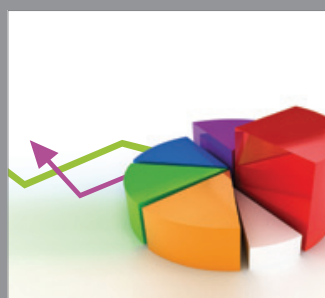

ournal of

Probability and Statistics

Promensencen
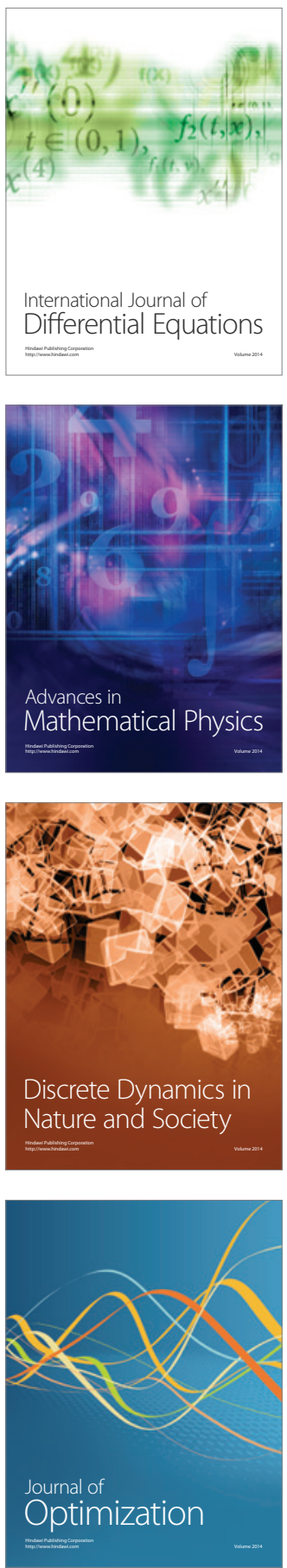